\title{
Analysis of the Effectiveness of the Application \\ of Fuzzy Voltage Regulation \\ in Distribution Networks
}

\author{
Vasiliy I. Panteleev, \\ Roman A. Petukhov* and Evgenia Yu. Sizganova \\ Siberian Federal University \\ 79 Svobodny, Krasnoyarsk, 660041, Russia
}

Received 26.01.2018, received in revised form 16.03.2018, accepted 24.06.2018

The article proposes a system for voltage regulation in distribution networks based on fuzzy logic. The fuzzy controller algorithm controls the operation of the on-load tap-changer of the power transformer and is responsible for connecting or disconnecting the batteries of static capacitors depending on the voltage level of the consumer.

Keywords: slow voltage changes, fuzzy logic, fuzzy regulation, distribution networks, reactive power.

Citation: Panteleev V.I., Petukhov R.A., Sizganova E.Yu. Analysis of the effectiveness of the application of fuzzy voltage regulation in distribution networks, J. Sib. Fed. Univ. Eng. technol., 2018, 11(5), 536-549. DOI: 10.17516/1999-494X-0052.

\section{Анализ эффективности применения \\ нечеткого регулирования уровня напряжения \\ в распределительных сетях}

\author{
В.И. Пантелеев, Р.А. Петухов, Е.Ю. Сизганова \\ Сибирский федеральный университет \\ Россия, 660041, Красноярск, пр. Свободный, 79
}

В статье предложена система регулирования напряжения в распределительных сетях на основе нечеткой логики. Нечеткий алгоритм регулятора управляет работой РПН силового трансформатора и отвечает за подключение или отключение батарей статических конденсаторов в зависимости от уровня напряжения у потребителя.

Ключевые слова: медленные изменения напряжения, нечеткая логика, нечеткое регулирование, распределительные сети, реактивная мошьность.

(c) Siberian Federal University. All rights reserved

* Corresponding author E-mail address: rom_pet1@mail.ru 


\section{Введение}

В районных и сельских распределительных сетях существует проблема несоответствия критериев качества электрической энергии нормам, которые установлены ГОСТ 32144-2013 «Электрическая энергия. Совместимость технических средств электромагнитная. Нормы качества электрической энергии в системах электроснабжения общего назначения». Наиболее часто потребители электрической энергии сталкиваются с низким уровнем установившегося напряжения, что приводит к уменьшению производительности исполнительных механизмов, снижению светового потока осветительных установок, погасанию дуговых и газоразрядных ламп, а в ряде случаев возможно нарушение технологического процесса со значительным экономическим ущербом.

Причиной этого является разветвленная конфигурация распределительных сетей, их большая протяженность, относительно малая установленная мощность потребителей и их территориальная разрозненность. Более того, существуют различные типы присоединения районных понизительных подстанций к энергосистеме высшего класса напряжения, имеющие свои достоинства и недостатки:

- радиальное - обеспечивает надежное электроснабжение, относительную простоту релейной защиты и автоматики. Однако требуются значительные затраты на сооружение РУ головной подстанции, строительство отдельной ВЛ или КЛ. Для питания районных понизительных подстанций применяется редко из-за дороговизны;

- магистральное - позволяет значительно уменьшить капиталовложения в строительство сети и РУ по сравнению с радиальной конфигурацией в ущерб надежности. Данный вид конфигурации сети получил наибольшее распространение для питания районных понизительных подстанций, несмотря на невысокую надежность, что допустимо для районных сетей из-за преобладания потребителей III категории надежности;

- магистральное с двухсторонним питанием - данный вид конфигурации позволяет повысить надежность электроснабжения районных понизительных подстанций, сочетая в себе основное достоинство магистральной конфигурации - дешевизну. Однако двухстороннее питание магистральной линии приводит, как правило, к увеличению ее протяженности и количества питаемых подстанций. Послеаварийные режимы данной конфигурации сети характеризуются особенно значительным падением напряжения на наиболее удаленных от центра питания подстанциях.

Кроме того, характерной особенностью питания районных и сельских понизительных подстанций является ограниченная мощность короткого замыкания сети высшего напряжения, высокое потребление реактивной мощности, что в еще большей степени оказывает отрицательное влияние на уровень установившегося напряжения и потери электрической энергии в максимальных и послеаварийных режимах работы сети.

В настоящее время способы поддержания заданного уровня напряжения у потребителей условно делятся на две группы [1]:

- устройства, изменяющие коэффициент трансформации;

- устройства, изменяющие потоки реактивной мощности.

Как правило, методы регулирования не всегда отвечают условиям быстродействия и эффективности. На современном этапе развития электроэнергетики особенно актуальным становится управление режимом работы распределительных сетей в режиме реального времени. 
Появление новых способов управления режимами работы распределительных сетей обусловлено тем, что при их эксплуатации возникает комплекс проблем, которые не всегда полностью поддаются решению традиционными методами. Все это заставляет создавать интеллектуальные системы, способные работать в условиях неопределенности и нечеткости исходной информации [2].

Решить возникающий комплекс проблем позволит система автоматического регулирования напряжения в распределительных сетях, основанная на алгоритмах нечеткой логики, преимущества которой по сравнению с другими системами управления доказаны в ряде работ.

Математическая модель. Для управления режимами работы сети с применением нечеткого регулятора требуется создание математической модели районной понизительной подстанции и распределительной сети. Структурная схема подстанции, сети и нечеткого регулятора представлена на рис. 1 .

Математическая модель районной распределительной сети в соответствии со структурной схемой включает в себя: внешнюю энергосистему (ЭС), районную понизительную подстанцию с силовыми понижающими трансформаторами (автотрансформаторами), батареями статических конденсаторов (БСК), системой нечеткого регулирования напряжения на шинах подстанции, линию электропередачи (ЛЭП), а также потребителя, представленного в виде узла комплексной электрической нагрузки.

Для создания системы нечеткого регулирования напряжения требуются следующие параметры режима: $i_{\ni c}, U_{\ni C}-$ ток, потребляемый из ЭС, и напряжение на стороне высшего напряжения (BH) подстанции; $i_{\Pi}, U_{\Pi}-$ ток и напряжение на зажимах электроприемника; $U_{\text {пс }}-$ напряжение на шинах низшего напряжения (НH) подстанции; $\mathrm{i}_{\text {Бск }}$ - ток БСК. Дополнительно требуется следующая информация: Т - положение отпайки РПН трансформатора; С - количество переключений устройства РПН за временной период; $\mathrm{N}$ - количество БСК в работе.

При моделировании принимаем, что режим работы сети симметричный, регулирующее воздействие путем изменения отпайки РПН и включения или отключения БСК осуществля-

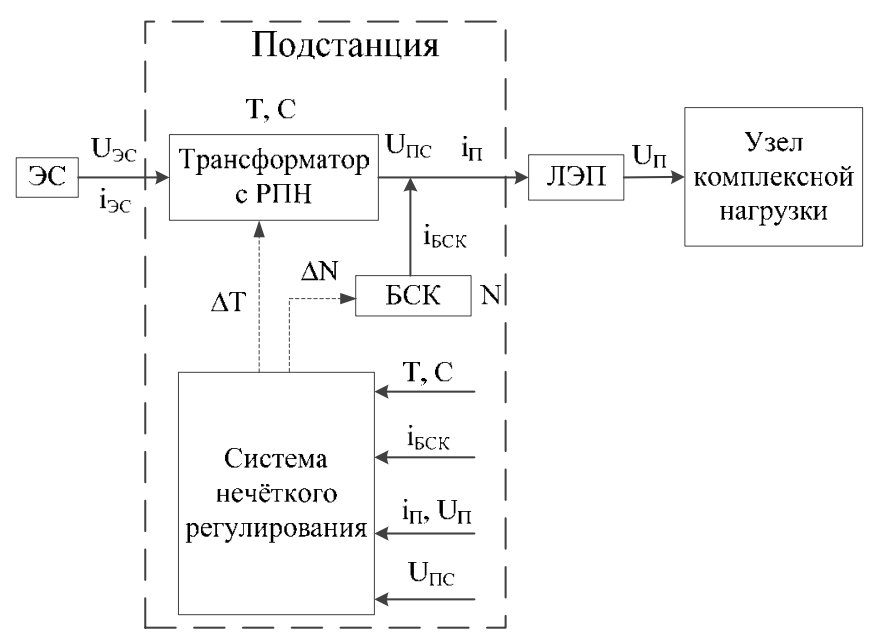

Рис. 1. Структурная схема

Fig. 1. Structure flowchart 
ется в каждой из трех фаз одновременно и симметрично, поэтому схемы замещения элементов распределительной сети (трансформатор, ЛЭП, комплексная нагрузка) представлены для одной фазы.

Схема замещения трансформатора изображена на рис. 2.

Математическая модель трансформатора примет вид

$$
\left\{\begin{array}{l}
\dot{U}_{2}=\left(\dot{U}_{1}-\left(\dot{I}_{1}-\dot{U}_{1}\left(G_{\mu}-j B_{\mu}\right)\right)\left(R_{T} \pm \Delta R_{T}+j X_{T} \pm \Delta X_{T}\right)\right) \cdot \frac{1}{n} \\
\dot{I}_{2}=\left(\dot{I}_{1}-\dot{U}_{1}\left(G_{\mu}-j B_{\mu}\right)\right) \cdot n
\end{array}\right.
$$

где $\dot{U}_{1}$ и $\dot{I}_{1}-$ напряжение, В, и ток, А, соответственно на стороне ВН районной понизительной подстанции; $R_{T}+j X_{T}$ - комплексное сопротивление одной фазы трансформатора, Ом; $\pm \Delta R_{T} \pm j \Delta X_{T}-$ комплексная добавка к сопротивлению фазы трансформатора, обусловленная изменением числа витков устройством РПН, Ом; $G_{\mu}-j B_{\mu}$ - комплексная проводимость ветви намагничивания трансформатора, См; $n$ - коэффициент трансформации; $\dot{U}_{2}$ и $\dot{I}_{2}$ - напряжение, $\mathrm{B}$, и ток, А, соответственно на стороне НН районной понизительной подстанции; $Z_{H}-$ комплексная нагрузка трансформатора.

Схему замещения БСК 10 кВ, располагаемых на подстанции, можно представить как регулируемый емкостный элемент (рис. 3), пренебрегая активными потерями из-за их малой доли относительно передаваемых мощностей.

Изменение емкости приводит к изменению генерируемой реактивной мощности, позволяя эффективно использовать возможности БСК. Изменение величины генерируемой реактивной мощности возможно осуществлять следующими способами:

1) ступенчатое включение/отключение секций БСК;

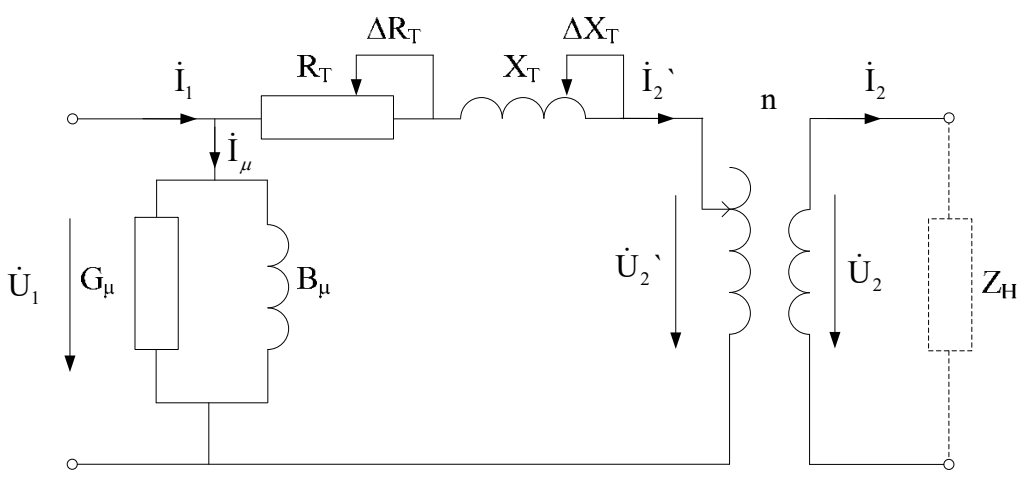

Рис. 2. Схема замещения трансформатора

Fig. 2. Transformer substitution circuit

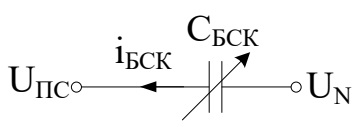

Рис. 3. Схема замещения БСК

Fig. 3. BSC substitution circuit 


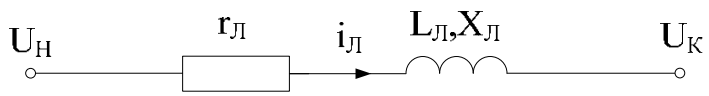

Рис. 4. Схема замещения ЛЭП

Fig. 4. Power line substitution circuit

2) использование тиристорного ключа;

3) включение/отключение индуктивности параллельно БСК.

Схема замещения ЛЭП выглядит следующим образом (рис. 4).

Математическая модель ЛЭП для установившегося режима такова:

$$
\dot{U}_{K}=\dot{U}_{H}-\dot{I}_{J} \cdot r_{J}-\dot{I}_{J} \cdot j X_{J},
$$

где $\dot{U}_{H}$ и $\dot{U}_{K}$ - напряжение в начале и конце линии соответственно, В; $\dot{I}_{Л}-$ ток в линии; $r_{J}-$ активное сопротивление ЛЭП, Ом; $X_{Л}=2 \pi f \cdot L_{Л}-$ индуктивное сопротивление линии, Ом.

Для мгновенных значений выражение примет вид

$$
U_{K}=U_{H}-i_{J} \cdot r_{J}-L_{J} \cdot \frac{d i_{J}}{d t}
$$

где $L_{Л}-$ индуктивность ЛЭП, Гн.

В модели ЛЭП не учитывается генерация реактивной мощности непосредственно линией, обусловленной емкостями между фазами, фазными проводами (жилами) и землей вследствие ее пренебрежимо малой величины по сравнению с передаваемыми мощностями по сети.

Схема замещения комплексной нагрузки. В районных и сельских распределительных сетях преобладает коммунально-бытовая нагрузка. Общепромышленная нагрузка представляет собой, как правило, небольшие производства, рассредоточенные географически. Представим всех потребителей электрической энергии в виде параллельно подключенных индуктивного, активного и емкостного элементов. Такое представление не учитывает возможность применения синхронных машин, работающих в режиме перевозбуждения для регулирования потока реактивной мощности (режим недовозбуждения в системах электроснабжения практически не используется из-за отсутствия необходимости потребления излишней реактивной мощности). Учесть такую возможность позволяет использование регулируемого емкостного элемента (рис. 5).

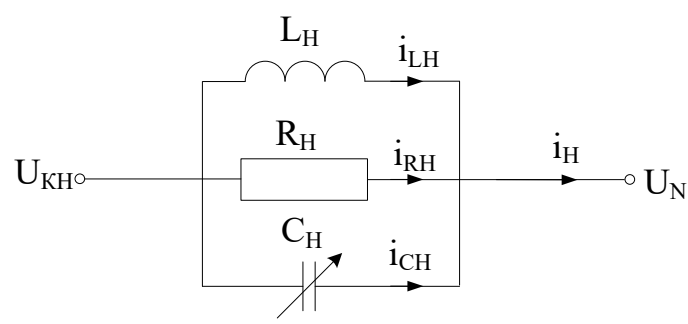

Рис. 5. Схема замещения комплексной нагрузки

Fig. 5. Complex load substitution circuit 
Математическая модель комплексной нагрузки:

$$
\left\{\begin{array}{l}
\dot{X}_{C H}=-j \frac{1}{2 \cdot \pi \cdot f \cdot C_{H}} \\
\dot{I}_{C H}=\frac{\dot{U}_{K H}-\dot{U}_{N}}{\dot{X}_{C H A}} \\
\dot{I}_{R H}=\frac{\dot{U}_{K H}-\dot{U}_{N}}{R_{\mathrm{H}}} \\
\dot{X}_{L \mathrm{H}}=j \cdot 2 \cdot \pi \cdot f \cdot L_{\mathrm{H}} \\
\dot{I}_{L A}=\frac{\dot{U}_{K H}-\dot{U}_{N}}{\dot{X}_{L H}} \\
\dot{I}_{\mathrm{H}}=\dot{I}_{L H}+\dot{I}_{C H}+\dot{I}_{R H}
\end{array}\right.
$$

Для мгновенных значений выражения примут следующий вид:

$$
\left\{\begin{array}{l}
i_{\mathrm{H}}=i_{L H}+i_{R H}+i_{C H} \\
i_{R H}=\frac{U_{K H}-U_{N}}{R_{\mathrm{H}}} \\
i_{C H}=C_{\mathrm{H}} \frac{d\left(U_{K H}-U_{N}\right)}{d t} \\
i_{L H}=i_{\mathrm{H}}-i_{R H}-i_{C H} \\
U_{K H}-U_{N}=L_{\mathrm{H}} \frac{d i_{L H}}{d t}
\end{array}\right.
$$

где $U_{K H}$ - напряжение на зажимах комплексной нагрузки относительно земли, В; $U_{N}-$ напряжение нейтрали относительно земли, $\mathrm{B} ; i_{L H} ; i_{R H} ; i_{C H}-$ полный ток комплексной нагрузки, токи индуктивной, активной и емкостной составляющих нагрузки, $\mathrm{A} ; L_{H}, R_{H}, C_{H}$ - индуктивная, активная и емкостная составляющие статической нагрузки, Гн, Ом, Ф.

Выражение для полного тока через узел комплексной нагрузки

$$
\dot{I}_{\mathrm{H}}=\left(\dot{U}_{K H}-\dot{U}_{N}\right)\left(\frac{1}{R_{H}}+j\left[2 \cdot \pi \cdot f \cdot L_{H}-\frac{1}{2 \cdot \pi \cdot f \cdot C_{H}}\right]\right) .
$$

Детальное математическое описание каждого элемента распределительной сети позволило создать полную математическую модель сети с возможностью расчета режимов ее работы.

Имитационное моделирование. Моделирование реализовано в пакете MATLAB Toolbox Fuzzy Logic [3].

Нечеткий алгоритм. Аппаратная реализация алгоритмов нечеткой логики позволяет учесть большое количество различных условий, оказывающих влияние на качество электрической энергии. Ключевым отличием нечеткого алгоритма от классических систем регулирования является относительная простота и высокое быстродействие.

Общие принципы нечеткого алгоритма сводятся к следующему: есть список нечетких правил и входные данные в виде сигналов, интерпретируемые устройством как нечеткие. Далее, применяя один из алгоритмов нечеткого вывода, аппаратная реализация которого осущест- 
вляется в виде контроллера, устройство обрабатывает входную информацию по созданным продукционным правилам и выдает управляющее воздействие по каждому правилу соответственно.

Схема нечеткого регулятора представлена тремя основными блоками (рис. 6). В первом блоке происходит фаззификация входных переменных, т.е. определяются их функции принадлежности к тому или иному нечеткому значению лингвистической переменной. Во втором блоке происходит нечеткий вывод, основанный на алгоритме Мамдани, формируются нечеткие значения выходных лингвистических переменных. Третий блок отвечает за дефаззификацию - получение определенных «четких» значений управляющих сигналов [4].

Нечеткое регулирование. Нечеткий логический регулятор, применяющий совместное использование РПН и БСК, позволяет решить задачи поддержания напряжения в сети и снижения потерь при передаче реактивной мощности. Кроме этого, разгружаются трансформаторы подстанции от передачи реактивной составляющей мощности в сеть низшего напряжения.

Количественно величина потерь мощности определяется по формуле, ВА:

$$
\Delta S=\frac{P^{2}+Q^{2}}{\left(U_{1}\right)^{2}} Z_{T P},
$$

где $P$ и $Q$ - активная, Вт, и реактивная, вар, мощность, пропускаемая через трансформатор; $U_{1}{ }^{\prime}-$ ВН трансформатора с учетом падения напряжения на активном и индуктивном сопротивлении обмоток по схеме замещения; $Z_{T P}$ - комплексное сопротивление трансформатора по схеме замещения, Ом.

Падение напряжения в сети, В:

$$
\Delta U=\frac{P_{H} R_{J}+Q_{H} X_{J}}{U_{L I I}}
$$

где $R_{J}$ и $X_{I}$ - активное и индуктивное сопротивление линии соответственно, Ом; $P_{H}$ и $Q_{H}$ активная, Вт, и реактивная, вар, мощность нагрузки соответственно; $U_{L I I}-$ напряжение, $\mathrm{B}$, на центре питания (шины НН подстанции).

Анализ непосредственных причин снижения напряжения у потребителей и в сети позволил определить входные (напряжение, реактивную мощность, положение отпайки и количество переключений РПН) и выходные (управление подключением и отключением БСК, управление переключением РПН) переменные для нечеткого контроллера (рис. 7).

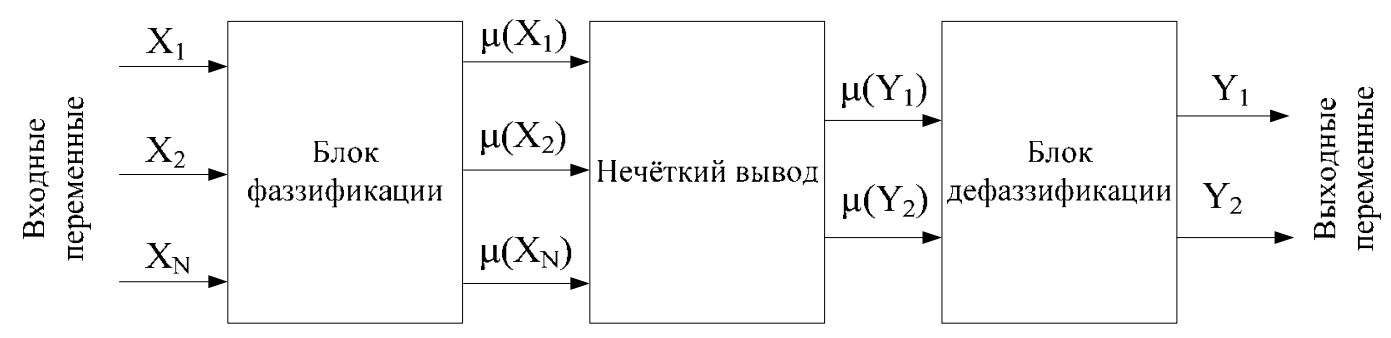

Рис. 6. Схема нечеткого регулятора

Fig. 6. Fuzzy controller circuit 


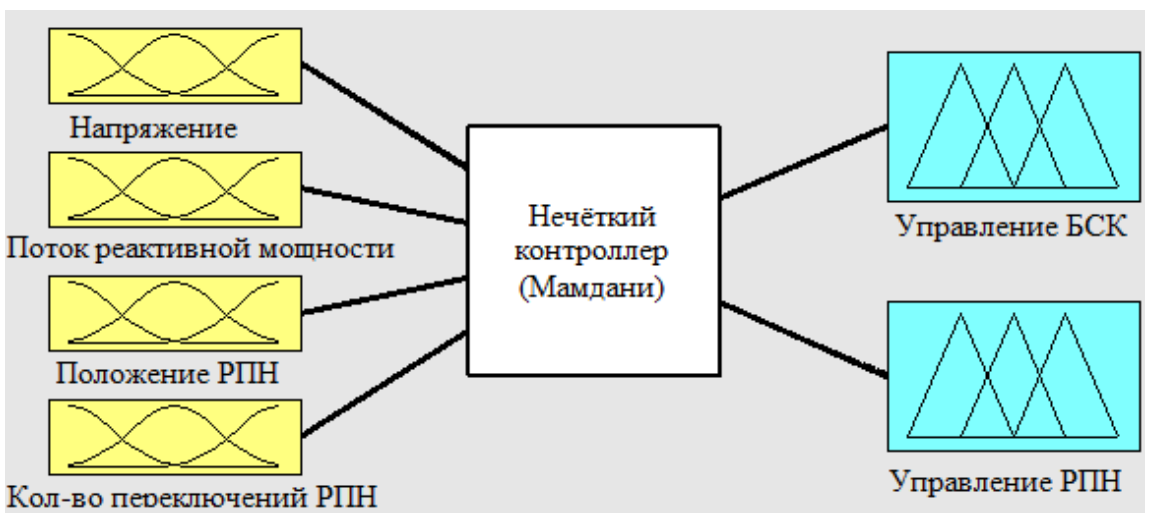

Рис. 7. Входные и выходные данные контроллера

Fig. 7. Controller Input and Output Data

С помощью РПН трансформатора напряжение нагрузки должно поддерживаться в нормируемых пределах. При этом поддерживаться оно должно не на вторичной обмотке трансформатора, а непосредственно у потребителя. Определить напряжение на зажимах потребителя можно, зная сопротивление линии и ток потребителя.

Значение напряжения, которое подается на вход нечеткого контроллера, соответствует необходимому уровню напряжения у потребителя, В,

$$
U_{\text {nотр }}=U_{\text {ЦІІ }}-I_{\text {потр }} \cdot Z_{Л},
$$

где $U_{\text {цा }}$ - напряжение на центре питания (шины НН подстанции); $I_{\text {потр }}$ - ток потребителя, А; $Z_{Л}-$ комплексное сопротивление линии до потребителя, Ом.

Текущее положение отпайки РПН как входного параметра необходимо для более «интеллектуального» поведения контроллера при подходе к конечному положению переключателя «анцапф». Входной параметр «количество переключений РПН в день» является важным и обоснованным, поскольку частое переключение устройства приводит к ускоренному износу и возрастанию вероятности выхода всего узла из строя.

В качестве нечеткого алгоритма был выбран алгоритм Мамдани. Это объясняется следующими факторами: простота программирования, наглядность и «понятность» всех составляющих алгоритма, возможность управления в режиме реального времени [4].

Функции принадлежности для определения степени истинности каждой предпосылки каждого правила имеют 3 вида: трапецеидальные, треугольные и Z-образные [4]. Системы уравнений, описывающих функции принадлежности:

$$
\begin{gathered}
\text { трапецеидальная: } \\
\mu(x)=\left\{\begin{array}{ll}
\text { Z-образная: } & \text { треугольная: } \\
\frac{x-a}{b-a}, & a<x<b \\
1, & b<x
\end{array} \quad \mu(x)=\left\{\begin{array}{ll}
1, & x<b, \\
\frac{c-x}{c-b}, & b<x<c \\
0, & c<x
\end{array} \quad \mu(x)= \begin{cases}0, & x<a, \\
\frac{x-a}{b-a}, & a<x<b, \\
\frac{c-x}{c-b}, & b<x<c \\
0, & c<x\end{cases} \right.\right.
\end{gathered}
$$


Для работы нечеткого контроллера были определены нечеткие значения входных и выходных лингвистических переменных.

\section{Bходные}

1. Напряжение на зажимах потребителя (рис. 8): низкое - Z-образная функция принадлежности; нормальное - трапецеидальная функция; высокое - Z-образная функция.

2. Реактивная мощность потребителя (рис. 9): низкая - Z-образная функция принадлежности; средняя - трапецеидальная функция; высокая - Z-образная функция.

3. Текущее положение отпайки РПН (рис. 10): отрицательное - Z-образная функция принадлежности; среднее - треугольная функция; положительное - Z-образная функция.

4. Количество переключений отпаек РПН (рис. 11): мало - Z-образная функция принадлежности; много - Z-образная функция.

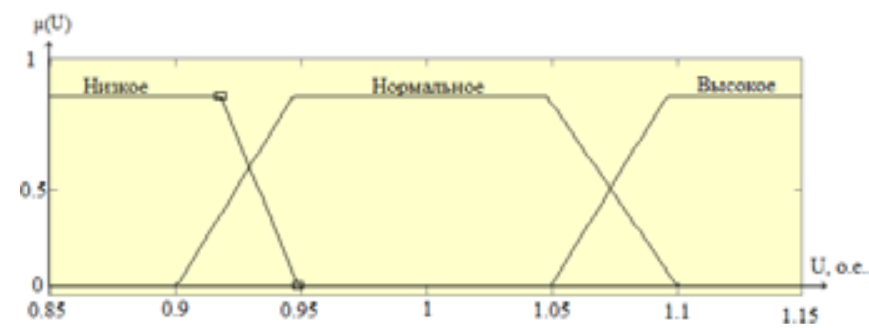

Рис. 8. Нечеткие значения лингвистической переменной «напряжение на зажимах потребителя»

Fig. 8. Fuzzy values of the linguistic variable "voltage at the terminals of the consumer"

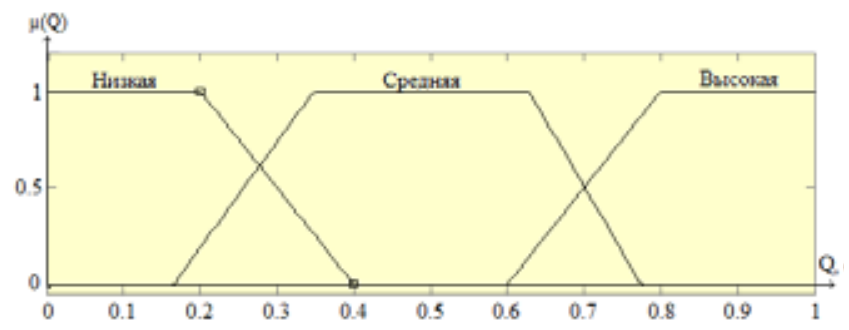

Рис. 9. Нечеткие значения лингвистической переменной «реактивная мощность потребителя»

Fig. 9. Fuzzy values of the linguistic variable "reactive power of the consumer"

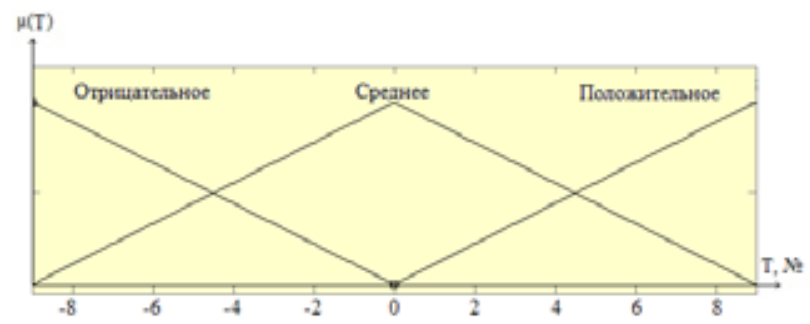

Рис. 10. Нечеткие значения лингвистической переменной «текущее положение отпайки РПН»

Fig. 10. Fuzzy values of the linguistic variable "current position of the tap-changer" 


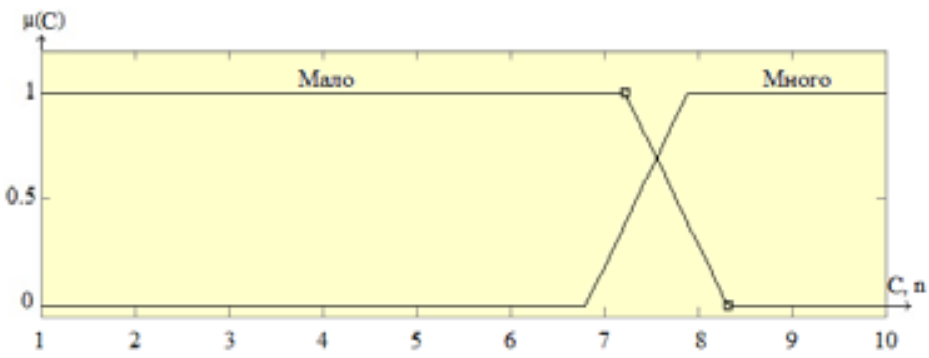

Рис. 11. Нечеткие значения лингвистической переменной «количество переключений отпаек РПН»

Fig. 11. Fuzzy values of the linguistic variable "number of tap-changer switchover"

\section{Bblxоднble}

1. Направление переключения отпайки РПН: вверх - трапецеидальная функция; не перемещать - трапецеидальная функция; вниз - трапецеидальная функция (рис. 12).

2. Коммутация БСК (рис. 13): 1-я ступень - трапецеидальная функция, 2-я ступень - трапецеидальная функция, 3-я ступень - трапецеидальная функция.

По лингвистическим переменным создана база продукционных правил (51 правило) для управления режимами работы рассматриваемой сети с привлечением знаний экспертов в предметной области:

- если напряжение у потребителя низкое, то положение отпайки РПН должно перемещаться вниз и/или должна быть подключена БСК в зависимости от уровня потребления реактивной мощности;

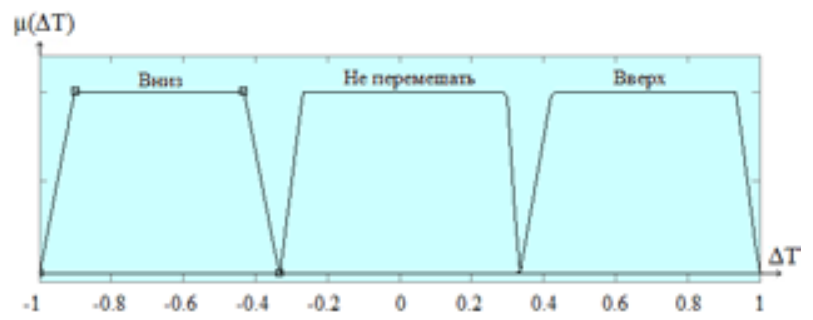

Рис. 12. Нечеткие значения лингвистической переменной «направление переключения отпайки РПН»

Fig. 12. Fuzzy values of the linguistic variable "direction of tap-changer switching"

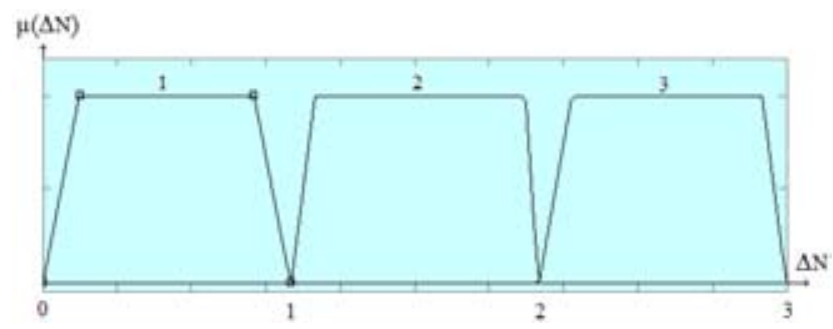

Рис. 13. Нечеткие значения лингвистической переменной «коммутация БСК»

Fig. 13. Fuzzy values of the linguistic variable "switching BSK" 


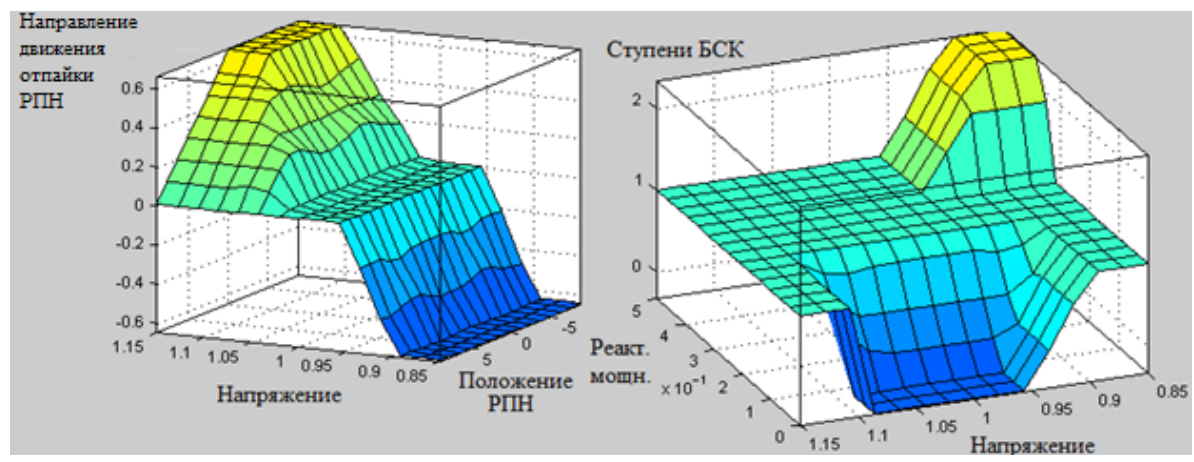

a)

б)

Рис. 14. Поверхности отклика: а - управление положением РПН; б - управление включением/ отключением БСК

Fig. 14. Response surfaces: a - control of the position of the on-load tap-changer; $\sigma$ - control of the on/off of the BSC

- если напряжение у потребителя высокое, то положение отпайки РПН должно перемещаться вверх и/или должна быть отключена БСК в зависимости от уровня потребления реактивной мощности;

- если количество переключений РПН подходит к критическому, то регулирование напряжения коммутацией БСК имеет преимущество.

По результатам моделирования были получены поверхности отклика (рис. 14), анализ которых показал, что разработанная нечеткая система регулирования принимает адекватное решение об управлении РПН трансформатора (рис. 14a) и коммутации БСК (рис. 14б). В области низких напряжений система принимает решение о перемещении отпайки РПН вниз, что в данной модели приведет к увеличению напряжения на шинах потребителя; в случае превышения напряжения выше номинального система принимает решение о переключении отпайки РПН в сторону понижения напряжения. При снижении напряжения и повышении потребления реактивной мощности система принимает решение о включении БСК и наоборот.

Таким образом, создана имитационная модель нечеткого логического контроллера, которая будет использована при моделировании участка электрической сети для анализа режимов ее работы и управления потоками реактивной мощности и напряжением.

Смоделированы режимы сброса и наброса нагрузки у потребителей, а также изменения напряжения с высокой стороны подстанции. Нагрузка в моделируемых ситуациях имела преимущественно активный и активно-индуктивный характер.

Моделирование участка электрической сети. Моделирование реализовано в пакете MATLAB Simulink SimPowerSystems [5]. Созданная модель состоит из следующих блоков (рис. 15):

«ЭС 110 кВ» - эквивалентная энергосистема напряжением 110 кВ, частотой 50 Гц;

«ТР» - эквивалентный силовой трансформатор мощностью 63 МВА, номинальным напряжением $110 / 10$ кB, схема соединения обмоток $\mathrm{Y}_{0} / \Delta$. На вход «Uр» подается импульсный сигнал на переключение отпайки РПН в следующее верхнее положение, на вход «Down» - сигнал на переключение РПН в следующее нижнее положение. C выхода «т» снимается информация о 


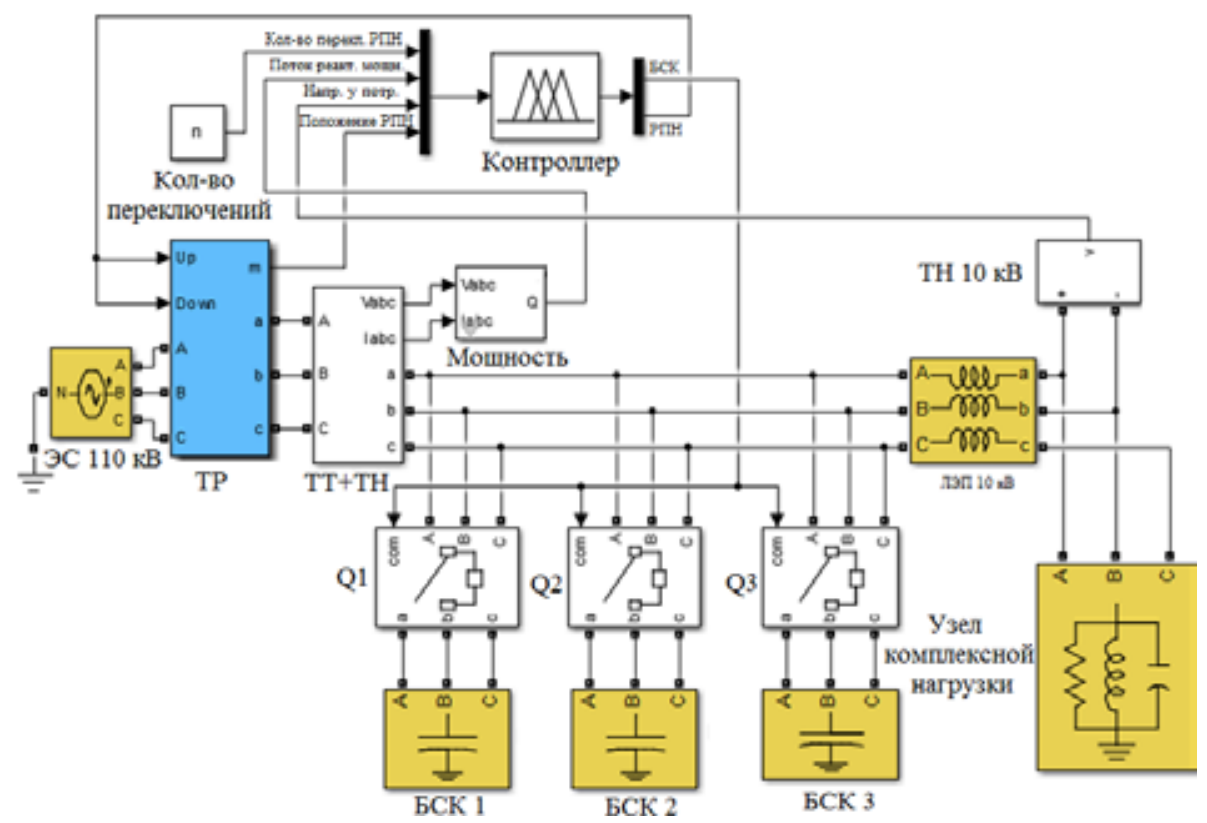

Рис. 15. Модель распределительной сети в среде Simulink

Fig. 15. Model of the distribution network in the Simulink environment

текущем положении отпайки РПН. «А», «В», «С», «a», «b», «c»- вводы ВН и НН по фазам соответственно;

«ТТ $+\mathrm{TH} »-$ комплект измерительных трансформаторов тока и напряжения. «А», «В», «С» и «a», «b», «c» - разъемы для подключения фазных проводов по фазам соответственно; «Vabc»разъем выходного сигнала фазных напряжений;

«Мощность»- блок вычисления потока реактивной мощности. На вход «V》 поступает сигнал об уровне напряжения; на вход «I» поступает сигнал о токе; с выхода «Q» снимается сигнал о величине потока реактивной мощности;

«Q1, Q2, Q3» - коммутационные аппараты в цепях БСК. «А», «В», «C» и «a», «b», «c»разъемы для подключения фазных проводов по фазам соответственно. На вход «т» подается импульсный сигнал для включения или выключения выключателя. Также в данной имитационной модели выключателя предусмотрен процесс разрыва цепи только при переходе напряжения через ноль, что имитирует процесс гашения дуги в реальном выключателе;

«БСК1, БСК2, БСК3» - батареи статических конденсаторов. Мощность одной ступени составляет 7,1 Мвар, схема соединения - «треугольник»;

«ЛЭП 10 кВ» - эквивалентная линия электропередачи 10 кВ, параметры задаются в зависимости от марки провода и длины линии;

«ТН 10 кВ»- измерительный трансформатор напряжения 10 кВ. Ко входам «+» и «-» подключаются две точки сети, между которыми требуется измерить напряжение (в созданной модели - линейное напряжение между фазами А и В). C выхода «V» снимается сигнал об уровне напряжения, который поступает на вход нечеткого контроллера;

«Узел комплексной нагрузки» - эквивалентная усредненная модель нагрузки;

$$
-547-
$$




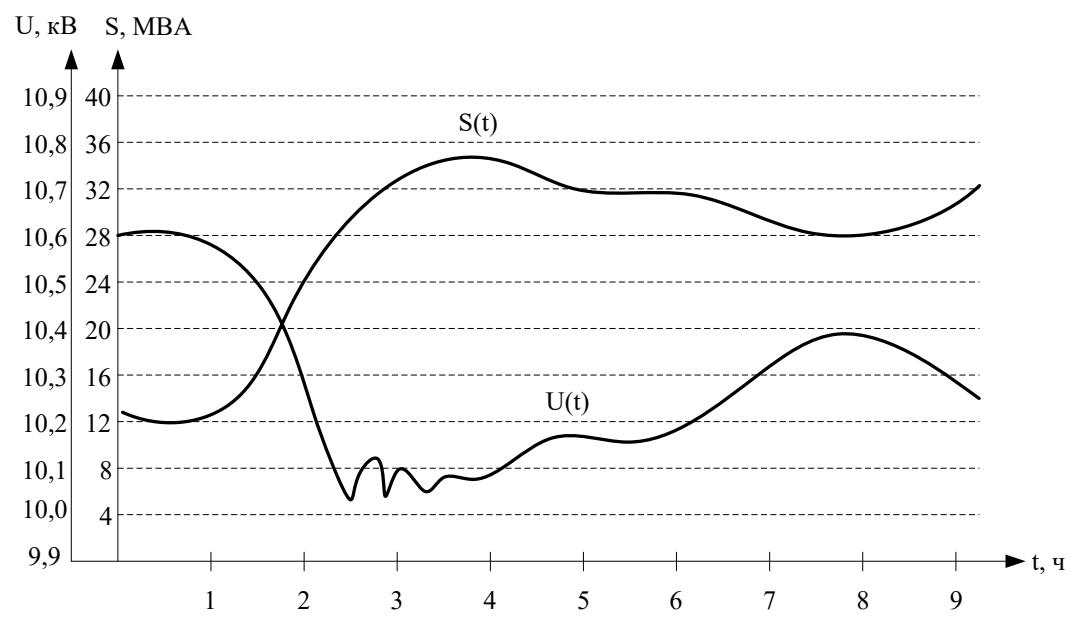

Рис. 16. График нагрузки и изменения напряжения

Fig. 16. Load graph and voltage changes

«Кол-во переключений» - блок, отвечающий за подсчет количества коммутаций аппаратами Q1, Q2, Q3, подает сигнал на вход нечеткого контроллера;

«Контроллер»- нечеткий контроллер.

По результатам моделирования были получены графики изменения нагрузки и напряжения (рис. 16).

В результате моделирования выяснилось, что нечеткий контроллер под управлением алгоритма Мамдани справляется с возложенными на него функциями. В режиме «реального времени» контроллер принимает решения об оптимальном положении отпайки РПН и количестве подключенных ступеней БСК в зависимости от потребляемой нагрузки. При этом показатель качества электроэнергии - медленные изменения напряжения электропитания - соответствует требованиям ГОСТ 32144-2013 «Электрическая энергия. Совместимость технических средств электромагнитная. Нормы качества электрической энергии в системах электроснабжения общего назначения».

\section{Выводы}

Внедрение нечеткого регулирования является современным способом автоматического управления напряжением, позволяющим с высокой точностью и быстродействием поддерживать напряжение на заданном уровне без участия оперативного персонала и отличающимся возможностью легкого расширения входных параметров, без глубокой переделки основ, при появлении необходимости в дополнительных функциональных требованиях.

На основании проведенных исследований можно утверждать, что предлагаемые нечеткие алгоритмы - достаточно мощная вычислительная процедура при неопределенности входной информации. 


\section{Список литературы}

[1] Веников В.А., Идельчик В.И., Лисеев М.С. Регулирование напряжения в электроэнергетических системах. М.: Энергоатомиздат, 1985. 214 с. [Venikov V.A., Idelchik V.I., Liseev M.S. Regulation of voltage in electric power systems. M.: Energoatomizdat, 1985. 214 p. (in Russian)]

[2] Богатырев Л.Л., Манусов В.З., Содномдорж Д. Математическое моделирование режимов электроэнергетических систем в условиях неопределенности. Улан Батор: Изд-во типографии МГТУ, 1999. 348 c. [Bogatyrev L.L., Manusov V.Z., Sodnomdorzh D. Mathematical modeling of modes of electric power systems in conditions of uncertainty. Ulaan Bator: Publishing house of MSTU, 1999. 348 p. (in Russian)]

[3] Леоненков А.В. Нечеткое моделирование в среде MATLAB и Fuzzy TECH: Основы теории нечетких множеств и нечеткой логики; Построение нечетких моделей в среде MATLAB Fuzzy Logic Toolbox; Создание проектов в пакете Fuzzy ТЕСН. СПб.: БХВ - Петербург, 2003. 736 c. [Leonenkov A.V. Fuzzy modeling in the environment of MATLAB and fuzzy TECH: Fundamentals of the theory of fuzzy sets and Fuzzy logic; Construction of fuzzy models in the environment MATLAB Fuzzy Logic Toolbox; Creating projects in the package Fuzzy TECH. St. Petersburg: BHV - Petersburg, 2003. 736 p. (in Russian)]

[4] Заде Л. Понятие лингвистической переменной и его применение к принятию приближенных решений. Пер. с англ. Н.И. Ринго. Под ред. Н.Н. Моисеева и С.А. Орловского. М.: Мир, 1976. 165 c. [Zadeh L. The concept of a linguistic variable and its application to the adoption of approximate solutions. Trans. with English. NI Ringo. Ed. N.N. Moiseeva and S.A. Orlovsky. Moscow: Mir, 1976. 165 p. (in Russian)]

[5] Kaszteny B., Rosolowski E., Izykowski J., Saha M.M., Hillstrom B. Fuzzy logic controller for on-load transformer tap changer. IEEE Transactions on Power Delivery, 1998, 13(January), 164-170. 